\title{
ALTERAÇÕES DE PROPRIEDADES FÍSICAS E HÍDRICAS DE UM LATOSSOLO VERMELHO DISTRÓFICO PELA ADSORÇÃO DE FÓSFORO(1)
}

\author{
R. B. SILVA(2), J . M. LIMA(3), M. S. DIAS J UNIOR ${ }^{(3)}$ \& F. A. M. SILVA(2)
}

\begin{abstract}
RESUMO
A elevada capacidade de adsorção de fósforo (P) é uma característica típica dos Latossolos oxídicos. O P é adsorvido principalmente por complexo de esfera interna que influencia o balanço de cargas, a dispersão de partículas e, conseqüentemente, a relação solo-água. 0 objetivo deste estudo foi avaliar o efeito da adsorção de $\mathrm{P}$ nas propriedades físicas e hídricas de amostras de um Latossolo Vermelho distrófico (L Vd), da região dos Campos da Mantiqueira (MG). Amostras deformadas e indeformadas, coletadas nas camadas de 0-0,03 e 0,27-0,30 m, foram saturadas em água pura ou em solução de $P$, com vistas em alcançar a capacidade máxima de adsorção de fósforo (CMAP). Posteriormente, avaliaram-se alterações no teor de argila dispersa em água, índice de floculação, porosidade, limites de consistência e curva de retenção deágua. A adsorção de $P$ aumentou a quantidade de argila dispersa e influiu na quantidade de macro e microporos, acarretando aumento dos limites de consistência no deslocamento da curva de retenção de água para valores mais elevados de umidade, assim como um acréscimo dos teores de água disponível.
\end{abstract}

Termos de indexação: teor de argila dispersa em água, curva de retenção de água, porosidade, limite de consistência, água disponível.

(1) Parte da Tese de Mestrado do primeiro autor, apresentada ao Departamento de Ciência do Solo, Universidade Federal de Lavras - DCS/UFLA. Projeto financiado pela CAPES. Recebida para publicação em abril de 2000 e publicada em julho de 2001.

(2) Doutorando do Departamento de Ciência do Solo, Universidade Federal de Lavras - UFLA. Caixa Postal 37, CEP 37200-000 Lavras (MG). Bolsista da CAPES. E-mail: rbsilva@ufla.br

(3) Professor Adjunto do Departamento de Ciência do Solo, UFLA. Bolsista do CNPq. E-mail: jmlima@ufla.br 


\title{
SUMMARY: CHANGES IN PHYSICAL AND HYDRIC PROPERTIES IN HAPLUDOX BY PHOSPHORUS SORPTION
}

\begin{abstract}
High phosphorus (P) sorption capacity is a typical featureof oxidic oxisols. P is mainly sorbed by inner-sphere complex affecting charge balance, particle dispersion and, consequently, water-soil relationship. The objective of this study was to evaluate the effect of $\mathrm{P}$ sorption on physical and hydric properties of samples of a Typic Hapludox, from Campos Mantiqueira Region (Minas Gerais, Brazil). Disturbed and undisturbed soil samples, from $0-0.03$ and $0.27-0.30 \mathrm{~m}$ depth, were saturated in pure water or $\mathrm{P}$ solution to reach the sorption capacity of each layer, followed by evaluation of clay dispersion, flocculation index, porosity, consistence limits, and water retention capacity. P sorption increased the content of dispersible clay and affected the amount of macro and micro pores, increasing the consistence limits and displacing the water retention curve to higher moisture values, causing an increase in available water content.
\end{abstract}

Index terms: content of dispersibleclay, water retention curve, porosity, consistence limits, available water.

\section{INTRODUÇÃO}

Parte da importância do fenômeno de adsorção de fósforo em solos deve-se ao fato de ser ele um dos ânions mais fortemente retidos, principalmenteem Latossolos (Vasconcellos, 1974; Bahia Filho, 1982). De acordo com Veith \& Sposito (1977), as reações de adsorção acontecem em dois estádios, um rápido, em que ocorrea adsorção propriamente dita, e um mais lento, a precipitação. O estádio rápido pode ser observado em superfícies de hematita e gibbsita (Kuo \& Lotse, 1974) e está, provavel mente, associado à alta energia de adsorção e à baixa saturação da superfície de adsorção. A adsorção do fósforo depende do tipo e da cristalinidade dos óxidos de ferro e alumínio, podendoser afetada pela matéria orgânica, principalmente, por esta competir com o íon fosfato pelos sítios de adsorção (Parfit et al., 1975).

Estudos desenvolvidos por Bahia Filho et al. (1983) Souza, et al. (1991) em Latossol os do Planalto Central, mostram que a goethita é o principal componente de óxido de ferro da fração argila responsável pela adsorção de fósforo. Segundo esses autores, isto decorre da substituição isomórfica do Fepel oAl. Esta substituição, de acordo com Resende (1976), provoca um desarranjo estrutural que diminui o tamanho das partículas, aumentando a área superficial específica e possibilitando, assim, maior adsorção de fósforo. A gi bbsita, se comparada à goethita, tem uma participação menos efetiva na adsorção de fósforo em solos oxídicos. Porém, em razão da sua mai or quantidade em al guns Latossolos, sua contribuição, em termos de adsor ção total, pode ultrapassar à de óxido de ferro (Curi et al., 1988; Mesquita Filho \& Torrent, 1993).

Como a maior parte das reações que envolvem óxidos de Fe e Al são dependentes de pH, o excesso de carga na superfície, positiva ou negativa, produz forças repulsivas entre partículas de cargas iguais, conseqüentemente, reduz a estabilidade de agregados eaumenta a dispersão de argila (Gillman, 1974).

A adsorção específica deíons deP forma complexos de esfera interna com grupos funcionais de superfície, particularmenteem sol os oxídicos, etorna mais negativa a superfície da partícula, desl ocando, deste modo, o ponto isoelétrico para um valor mais baixo (Hingston et al., 1974; Sawhney, 1974; Sposito, 1989). Em sol os com balanço de carga líquida positiva (isto é, solos com valores de $\mathrm{pH}$ menores que os do PCZ), a exemplo da maioria dos Latossolos, a adsorção de fosfato aumenta a quantidade de cargas negativas e diminui o excedente de cargas positivas, aumentando, assim, a atração el etrostática entreas partículas e, conseqüentemente, diminuindo a dispersão (aumento da floculação) até o ponto em que esse balanço é zero (máxima floculação).

A partir desse ponto, a adsorção de fósforo altera a quantidade de argila dispersa em água (ADA), a qual favorece a desagregação (Lima et al., 2000). Essa alteração afeta a quantidade de macro e mi croporos, estabilidade deagregados, dispersão de partículas, com efeito sobre os limites de consistência e pressão de preconsolidação (Silva et al., 1999).

Portanto, a exemplo de outros íons (E L-Swaify, 1975; Valle J únior, 1995), o íon fosfato pode reduzir o efeito cimentante dos óxidos de Fe eAl e alterar o estado de agregação, comprometendo as propriedades físicas e hídricas do solo e, conseqüentemente, influenciar o comportamento de água no solo.

Este trabalho teve por objetivo avaliar as alterações deal gumas propriedades físicas e hídricas de um Latossolo Vermelho distrófico (LVd), fase cerrado, sob o efeito da adsorção de fósforo. 


\section{MATERIAL E MÉTODOS}

Neste estudo, foram utilizadas amostras indeformadas $(0,0652$ m de diâmetro $\times 0,0254$ m dealtura) e deformadas das camadas de 0-0,03 e 0,27-0,30 m de um LVd, fase cerrado, localizado nos Campos da Mantiqueira (MG), entre $21^{\circ} 15^{\prime}$ e $21^{\circ} 50^{\prime} \mathrm{S} 44^{\circ} 15^{\prime}$ e $44^{\circ} 45^{\prime} \mathrm{W}$.

No quadro 1, está apresentada a caracterização química e mineralógica de amostras indeformadas de sol o das camadas estudadas. Os teores de óxidos extraídos pelo ataque sulfúrico, fósforo disponível, carbono orgânico e valores de $\mathrm{pH}$ foram determinados segundo Vettori (1969) e EMBRAPA (1997). Os teores de caulinita e gibbsita foram quantificados por meio deanál ise térmica diferencial (ATD), após desferrificação da argila pelo método do ditionito-citrato-bicarbonato (DCB), conforme Mehra \& J ackson (1960). O ponto decarga zero (PCZ) foi determinado indiretamente pela expressão: $2 \mathrm{pH}_{\mathrm{KCl}}-\mathrm{pH}_{\mathrm{H}_{2} \mathrm{O}}$, sugerida por Keng \& Uehara (1974).

A capacidade máxima de adsorção de fósforo (CMAP) foi determinada com base no $P$ remanescente (Alvarez \& Fonseca, 1990), seguindo o procedimento metodol ógico de Olsen $\&$ Watanabe (1957). Os valores de adsorção encontrados foram ajustados à equação de Langmuir (Rajan \& Fox, 1975) que, depois de linearizada, originou a energia de adsorção, K (b/a) e a CMAP (1/b), como mostra o quadro 2.

Para avaliar o efeito da adsorção de $\mathrm{P}$ sobre as propriedades físicas ou hídricas, duas condições foram atendidas: com P esem P. Na primeira, parte das amostras foi tratada com solução de $\mathrm{P}$, (fonte: $\mathrm{KH}_{2} \mathrm{PO}_{4}$ ) suficiente para se atingir CMAP, durante as etapas de umedecimento ( $24 \mathrm{~h}$ ) ou de saturação (48 h), conforme o atributo aval iado (Quadro 2). Na segunda condição, o restante das amostras recebeu apenas água destilada.

A textura foi avaliada com base no método de Gee \& Bauder (1986). Nos tratamentos que receberam $\mathrm{P}$, foram aplicadas concentrações equivalentes a CMAP para $10 \mathrm{~g}$ de terra fina seca em estufa (TFSE) da amostra. Os índices de floculação (IF) foram calculados com base nas expressões: (a) amostras sem fósforo: IF $=[($ AT ADA $/ A T$ ] * 1.000; e (b) amostras com fósforo: I $F_{\mathrm{P}}=$ [(AT-ADP)/AT] * 1000, sendo IF $\mathrm{P}$ o índice de floculação da amostra com fósforo; IF o índice de floculação sem fósforo; ADP argila dispersa em solução de P; AT é a argila total e ADA é a argila dispersa em água.

\section{Quadro 1. Caracterização química e mineralógica do Latossolo Vermelho distrófico}

\begin{tabular}{|c|c|c|c|c|c|c|c|c|c|c|c|c|c|c|c|}
\hline \multirow[b]{2}{*}{ Camada } & \multicolumn{5}{|c|}{ Ataque sulfúrico } & \multirow[b]{2}{*}{$\mathbf{F e}_{\mathrm{d}}$} & \multirow[b]{2}{*}{ Feo } & \multicolumn{2}{|c|}{ Rel. molec. } & \multirow[b]{2}{*}{ M.O. ${ }^{(1)}$} & \multicolumn{2}{|c|}{ pH } & \multirow[b]{2}{*}{$\mathbf{P C Z} Z^{(2)}$} & \multirow[b]{2}{*}{$\mathrm{Ct}^{(3)}$} & \multirow[b]{2}{*}{$\mathbf{G} \mathbf{b}^{(4)}$} \\
\hline & $\mathrm{SiO}_{2}$ & $\mathrm{Al}_{2} \mathrm{O}_{3}$ & $\mathrm{Fe}_{2} \mathrm{O}_{3}$ & $\mathrm{TiO}_{2}$ & $\mathbf{P}_{2} \mathrm{O}_{5}$ & & & $\mathbf{K i}$ & $\mathbf{K r}$ & & Água & $\mathrm{KCl}$ & & & \\
\hline $\mathrm{m}$ & $\underline{+}$ & 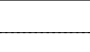 & 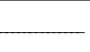 & $\mathrm{kg}^{-1}$ & 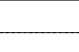 & 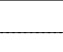 & - & & & $\mathrm{g} \mathrm{kg}^{-1}$ & & & & & $g^{-1}$ \\
\hline $0-0,03$ & 136 & 316 & 156 & 6,9 & 1,02 & 63 & 1,9 & 0,73 & 0,56 & 38 & 5,2 & 4,4 & 3,6 & 240 & 400 \\
\hline $0,27-0,30$ & 137 & 306 & 163 & 8,5 & 0,81 & 59 & 1,6 & 0,76 & 0,57 & 29 & 5,3 & 4,6 & 3,9 & 250 & 380 \\
\hline
\end{tabular}

(1) Matéria orgânica. ${ }^{(2)}$ Ponto de carga zero. ${ }^{(3)}$ Caulinita. ${ }^{(4)}$ Gibbsita.

\section{Quadro 2. Quantidades de $\mathbf{P}$ das respectivas massas de solo, utilizadas nas análises das propriedades avaliadas, considerando a capaci dade máxi ma de adsorção de P (CMAP) de amostras de duas camadas do solo}

\begin{tabular}{|c|c|c|c|c|c|c|c|c|}
\hline \multirow{4}{*}{ Camada } & \multirow{4}{*}{\multicolumn{2}{|c|}{ Equação }} & \multirow{4}{*}{$\mathbf{K}$} & \multirow{4}{*}{ CMAP } & \multirow{2}{*}{\multicolumn{2}{|c|}{$\begin{array}{c}\text { Amostra indeformada }{ }^{(1)} \\
\text { Curva de retenção } \\
(2)\end{array}$}} & \multicolumn{2}{|c|}{ Amostra deformada $^{(1)}$} \\
\hline & & & & & & & Consistência & Textura \\
\hline & & & & & \multirow{2}{*}{$P$ cilind $^{-1}{ }^{-1}$} & \multirow[t]{2}{*}{ P total ${ }^{(3)}$} & \multicolumn{2}{|c|}{ Massa de solo, $g$} \\
\hline & & & & & & & 500 & 10 \\
\hline $\mathrm{m}$ & & & & 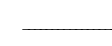 & -1 & $\mathrm{P}, \mathrm{mg} \mathrm{kg}^{-1}$ & & - \\
\hline $0-0,03$ & $Y=0,000769 x+0,00103$ & $\mathrm{R}^{2}=0,99$ & 0,74 & 1.299 & 149,24 & $2.238,63$ & 838,69 & 16,76 \\
\hline $0,27-0,30$ & $Y=0,000641 x+0,000482$ & $\mathrm{R}^{2}=0,99$ & 1,33 & 1.559 & 253,69 & $3.805,35$ & $1.218,85$ & 23,22 \\
\hline
\end{tabular}

(1) A quantidade de $\mathrm{P}$ fornecida para que se atingisse a CMAP nos parâmetros avaliados dependeu da umidade e densidade do material de solo de cada camada. ${ }^{(2)}$ Nos anéis utilizados para determinação da curva de retenção, determinaram-se também a densidade do solo inicial, a macro e a microporosidade. ${ }^{(3)}$ Fósforo total aplicado em 15 cilindros, número utilizado por tratamento. 
O volume de macro e microporos foi determinado em amostras indeformadas na presença e ausência de fósforo, conforme EMBRAPA (1997). Os limites deliquidez (LL) ede plasticidade foram determinados segundo Sowers (1965), e o limite de contração (LC) de acordo com Bowles (1986).

A curva de retenção deágua foi obtida por secagem, com base em Klute (1986). As amostras, depois de saturadas por $48 \mathrm{~h}$ em solução de fósforo (conforme a CMAP) ou em água destilada, foram levadas à mesa detensão $(0,002 ; 0,004 ; 0,006 ; 0,008 ; 0,01 \mathrm{MPa})$ e, posteriormente, às câmaras de pressão $(0,033 ; 0,1$; 0,5 e 1,5 MPa), até que o teor de água equilibrasse. Os val ores de umi dade e sucção foram ajustados ao modelo de Genuchten (1980) $\theta=\theta_{r}+\left(\theta_{s}-\theta_{r}\right) /[(1+$ $\left.(\alpha . h)^{\mathrm{n}}\right]^{\mathrm{m}}$, em que $\theta$ é a umidade volumétrica à base de volume; $\theta_{s}$ e $\theta_{r}$ são as umidades volumétricas de saturação e residual, respectivamente; h éa tensão em c.c.a., e $\alpha$, men são parâmetros deajuste domodelo.

O experimento foi instalado no delineamento em blocos completos ao acaso, sendo as análises de variância e testes de médias realizados por profundidade amostrada. As variáveis qualitativas foram analisadas a partir do software Sanest (Zonta et al., 1984) e as quantitativas, particularmente as curvas de retenção de água, foram ajustadas por meio do model o de Genuchten (1980), utilizando um programa para ajuste da curva de retenção de água proposto por Dourado Neto et al. (1990).

\section{RESULTADOS E DISCUSSÃO}

Os efeitos da adsor ção de fósforo com alterações das propriedades físicas (teor de argila dispersa, índice defloculação, densidade do sol o inicial emacro e microporosidade) são apresentados no quadro 3.

A adição deP alterou os teores deargilas dispersas, reduzindo significativamente o índice de floculação em ambas as profundidades estudadas. A dispersão, além de diminuir os valores de densidade inicial, contribuiu para reduzir e el evar significativamente os teores de macro e microporos, respectivamente. A alteração destas propriedades deveu-se, provavelmente, à diminuição do efeito cimentante dos óxidos, que, de acordo com Shainberg \& Letey (1984), pode alterar a geometria dos poros e alterar a condutividade hidráulica. A maior tendência de respostas à adsorção de $P$ das propriedades aval iadas na camada subsuperficial $(0,27-0,30 \mathrm{~m})$ deveu-seao menor teor de matéria orgânica eaos maiores teores de gibbsita (Quadro 1), o que favoreceu a maior adsorção de fósforo e, portanto, o maior efeito dispersivo, em conseqüência do desbalanço de cargas na superfície dos colóides.

Vale ressaltar que, neste solo, os valores de $\mathrm{PCZ}$ estão abaixo dos valores de pH (Quadro 1), e a adsorção deP el evou a quantidade decarga negativa, conferindo-lhe um balanço de carga negativo.

A adsorção de fosfato elevou os $L L, L P$ e LC, propiciando aumentos dos índices de plasticidade (IP) e da faixa de friabilidade (F F) (Quadro 4). I sto sedeveu, provavel mente, à mai or superfícieespeáfica, que certamente influenciou interação solo-água, elevando, por sua vez, a retenção de água (microporosidade), como mostra o quadro 1 . 0 deslocamento do IP e da FF para teores mais el evados de água, além de dificultar o preparo deste solo, diminui a capacidade de suporte, susceptibilizando-o à compactação (Dias J unior, 1994; Dias J unior \& Pierce, 1996; Kondo \& Dias J unior, 1999; Silva et al., 1999). Vale ressaltar que as respostas obtidas neste estudo basearam-se na CMAP, concentrações de P que, certamente, são muito superiores às fosfatagens ou adubações corretivas usadas rotineiramente.

Outro efeito da adsorção deP sobre propriedades que governam a interação solo-água pode ser visto no quadro 5 e nas figuras 1 e 2. O P influiu nos parâmetros de ajustes descritos pelo modelo de Genuchten (1980) e elevou as suas respectivas umidades vol umétricas residuais $\left(\theta_{\mathrm{r}}\right)$, de saturação $\left(\theta_{s}\right)$, resultando em al terações no comportamento das curvas de retenção de água (Figura 1).

Quadro 3. Quantidades de micro e macroporos, argila dispersa, índice de floculação e densidade inicial em duas camadas do solo, com e sem fósforo

\begin{tabular}{|c|c|c|c|c|c|c|c|c|c|c|c|}
\hline \multirow{3}{*}{ Camada } & & & & \multirow{2}{*}{\multicolumn{2}{|c|}{$\begin{array}{c}\text { Índice de } \\
\text { floculaçãao }\end{array}$}} & \multirow{2}{*}{\multicolumn{2}{|c|}{ Densidade inicial }} & \multicolumn{4}{|c|}{ Porosidade } \\
\hline & \multicolumn{3}{|c|}{ Teor de argila } & & & & & \multicolumn{2}{|c|}{ Macro } & \multicolumn{2}{|c|}{ Micro } \\
\hline & Total $^{(1)}$ & Água $^{(2)}$ & $\mathbf{P}^{(3)}$ & Água & $\mathbf{P}$ & Sem $P$ & $\operatorname{Com} \mathbf{P}$ & Sem $\mathbf{P}$ & $\operatorname{Com} \mathbf{P}$ & Sem $P$ & Com P \\
\hline $\mathrm{m}$ & & 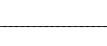 & $\mathrm{kg}^{-1}$ & 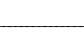 & - & $\mathrm{M}_{\xi}$ & $\mathrm{A}^{-3}$ & 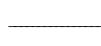 & $\mathrm{m}^{3}$ & -3 & {[} \\
\hline $0-0,03$ & $663 \mathrm{a}$ & $150 \mathrm{c}$ & $320 \mathrm{~b}$ & $773 \mathrm{a}$ & $517 \mathrm{~b}$ & $1,01 \pm 0,04$ & $0,99 \pm 0,04$ & $0,222 \mathrm{a}$ & $0,184 \mathrm{~b}$ & $0,381 \mathrm{~b}$ & $0,415 \mathrm{a}$ \\
\hline $0,27-0,30$ & $685 \mathrm{a}$ & $185 \mathrm{c}$ & $410 \mathrm{~b}$ & $729 \mathrm{a}$ & $401 \mathrm{~b}$ & $1,06 \pm 0,02$ & $1,05 \pm 0,04$ & $0,232 \mathrm{a}$ & $0,210 \mathrm{~b}$ & $0,386 \mathrm{~b}$ & $0,411 \mathrm{a}$ \\
\hline
\end{tabular}

(1) Teor de argila dispersa em solução hidróxido de sódio. ${ }^{(2)}$ teor de argila dispersa em água. ${ }^{(3)}$ Teor de argila dispersa em solução de fósforo. Letras minúsculas, na linha, comparam teor de argila dispersa, índice de floculação e porosidade na condição com e sem $\mathrm{P}$, a $5 \%$ de probabilidade pelo teste de Tukey. 
Verificou-se maior retenção de água para as amostras saturadas com solução de P. Embora a camada de 0-0,03 m (Figura 1a) tenha possibilitado maior retenção de água, principalmente a baixas tensões, foi o material da camada de 0,27-0,30 m (Figura 1b) que apresentou maior efeito da adsorção defósforo, como mostra o desl ocamento da curva das amostras saturadas com $\mathrm{P}$ em relação à curva das amostras saturadas apenas com água. Tal fato pode ser atribuído aos menores e maiores teores de matéria orgânica e de gibssita, respectivamente, (Quadro 1), conferindo-Ihe maior CMAP e energia de adsorção (K) (Quadro 2), concordando, portanto com Kuo \& Lotse (1974).

Assim, se, por um lado, a matéria orgânica concorre diretamente com o ânion fosfato pelos mesmos sítios de adsorção da superfície dos óxidos de ferro ealumínio, por outro, favorece uma melhor estruturação (Sawhney, 1974; Parfitt et al., 1975; Sposito, 1989; Lima 1995), justificando, assim, um menor efeito da adsorção e mai or retenção de água na camada superficial (Figura 1a). O efeito da adsorção de $\mathrm{P}$ pode ainda ser mais bem visualizado nas figuras $2 a$ e $2 b$, em que é mostrado o efeito isolado da adsorção de $\mathrm{P}$ sobre a retenção de água em cada camada. Observa-se que na condição com $P$ (Figura 2b), comparativamente à condição sem $\mathrm{P}$ (Figura 2a), houve maior deslocamento das curvas características para umidades mais el evadas, sendo este desl ocamento mais expressivo, principalmente, em amostras da camada de 0,27-0,30 m.

$A$ adsorção de $P$ alterou também a água disponível (AD), ou seja, o teor deágua retido entre a capacidade de campo (0,01 MPa) e o ponto de murcha permanente (1,5 $\mathrm{MPa})$, como mostram a figura 3 e o quadro 5 . Em materiais de solo da camada de 0-0,03 m (Figura 3a), apesar da maior retenção de água, foi desprezível o aumento da $A D$ $\left(0,01 \mathrm{~m}^{3} \mathrm{~m}^{-3}\right)$, o que pode ser constatado pelo paral el ismo das curvas das condições estudadas. $\mathrm{Na}$ camada de 0,27-0,30 m (Figura 3b), a adsorção de P el evou cerca de 2,7 vezes o teor de AD em relação à condição sem P. O teor de água referente à capacidade de campo parece ter sido o responsável por esse incremento, já que a diferença entre os teores de água relativa à capacidade decampo entre as condições com e sem $\mathrm{P}$ dessa camada $(0,48$ e $0,28 \mathrm{~m}^{3} \mathrm{~m}^{-3}$ ) mostrou-se muito superior à diferença entre os teores de 0,42 e 0,35 $\mathrm{m}^{3} \mathrm{~m}^{-3}$ da camada de $0-0,03 \mathrm{~m}$.

Quadro 4. Limites de liquidez (LL), limites de plasticidade (LP), limites de contração (LC), índice de plasticidade e faixas de friabilidade em duas camadas do solo

\begin{tabular}{|c|c|c|c|c|c|c|}
\hline \multirow{2}{*}{ Camada } & \multirow{2}{*}{ Condição de saturação } & \multicolumn{3}{|c|}{ Limite de consistência } & \multirow{2}{*}{ Faixa de friabilidade } & \multirow{2}{*}{ Índice plástico } \\
\hline & & $\mathbf{L L}$ & LP & LC & & \\
\hline $\mathrm{m}$ & & 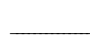 & 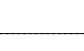 & 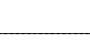 & $\mathrm{kg} \mathrm{kg}^{-1}$ & - \\
\hline $0-0,03$ & $\begin{array}{l}\text { Água pura } \\
\text { Solução de P }\end{array}$ & $\begin{array}{l}0,44 \\
0,62\end{array}$ & $\begin{array}{l}0,33 \\
0,39\end{array}$ & $\begin{array}{l}0,22 \\
0,26\end{array}$ & $\begin{array}{l}0,11 \\
0,13\end{array}$ & $\begin{array}{l}0,11 \\
0,23\end{array}$ \\
\hline $0,27-0,30$ & $\begin{array}{l}\text { Água pura } \\
\text { Solução de P }\end{array}$ & $\begin{array}{l}0,40 \\
0,57\end{array}$ & $\begin{array}{l}0,25 \\
0,35\end{array}$ & $\begin{array}{l}0,16 \\
0,22\end{array}$ & $\begin{array}{l}0,09 \\
0,13\end{array}$ & $\begin{array}{l}0,15 \\
0,22\end{array}$ \\
\hline
\end{tabular}

Quadro 5. Água disponível, teores deágua referentes à capacidade de campo, ponto de murcha permanente e equação de Genuchten ajustada para as condições com e sem P em duas camadas do solo (q = umidade à base de volume; $\theta_{r}=$ umidade residual; $\theta_{s}=$ umidade de saturação; $h=$ tensão em c.c.a.; $\alpha$, m e $\mathbf{n}=$ parâmetros empíricos)

\begin{tabular}{|c|c|c|c|c|c|}
\hline Camada & Condição & $\theta=\theta r+\left(\theta_{\theta s^{-}} \theta_{\rho}\right) /\left[1+(\alpha \cdot h)^{n}\right]^{m}$ & $\theta c c^{(1)}$ & $\theta$ PMP ${ }^{(2)}$ & $A D^{(3)}$ \\
\hline $\mathrm{m}$ & & & 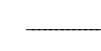 & $m^{3} m^{-3}$ & - \\
\hline $0-0,03$ & $\begin{array}{l}\text { Água pura } \\
\text { Solução de P }\end{array}$ & $\begin{array}{l}\theta=0,24+(0,52-0,24) /\left[1+(0,085 \cdot h)^{1,411}\right]^{0,291} \\
\theta=0,28+(0,58-0,28) /\left[1+(0,119 \cdot h)^{1,317}\right]^{0,241}\end{array}$ & $\begin{array}{l}0,35 \\
0,42\end{array}$ & $\begin{array}{l}0,25 \\
0,31\end{array}$ & $\begin{array}{l}0,10 \\
0,11\end{array}$ \\
\hline $0,27-0,30$ & $\begin{array}{l}\text { Água pura } \\
\text { Solução de P }\end{array}$ & $\begin{array}{l}\theta=0,24+(0,49-0,24) /\left[1+(0,099 \cdot h)^{1,416}\right]^{0,294} \\
\theta=0,28+(0,52-0,28) /\left[1+(0,069 \cdot h)^{1,279}\right]^{0,218}\end{array}$ & $\begin{array}{l}0,28 \\
0,42\end{array}$ & $\begin{array}{l}0,24 \\
0,32\end{array}$ & $\begin{array}{l}0,04 \\
0,10\end{array}$ \\
\hline
\end{tabular}

(1) Umidade referente à capacidade de campo (0,01 MPa). (2) U midade referente ao ponto de murcha permanente (1,5 MPa). ${ }^{(3)}$ Água disponível $=\theta_{\mathrm{CC}}-\theta_{\mathrm{PMP}}$. 


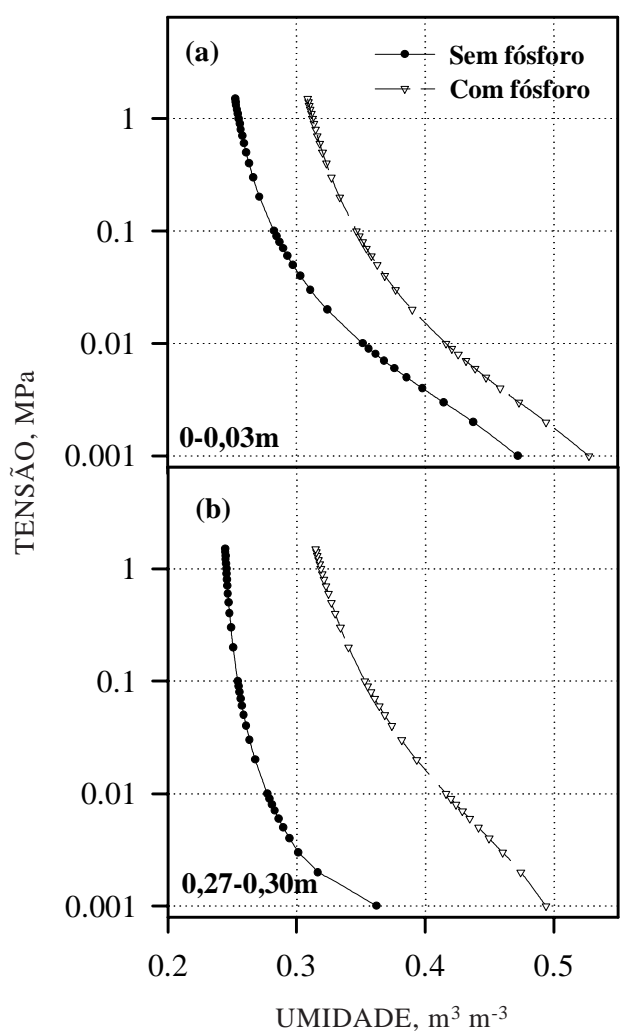

Figura 1. Curvas de retenção de água de material de solo, com e sem P, em duas camadas do solo.

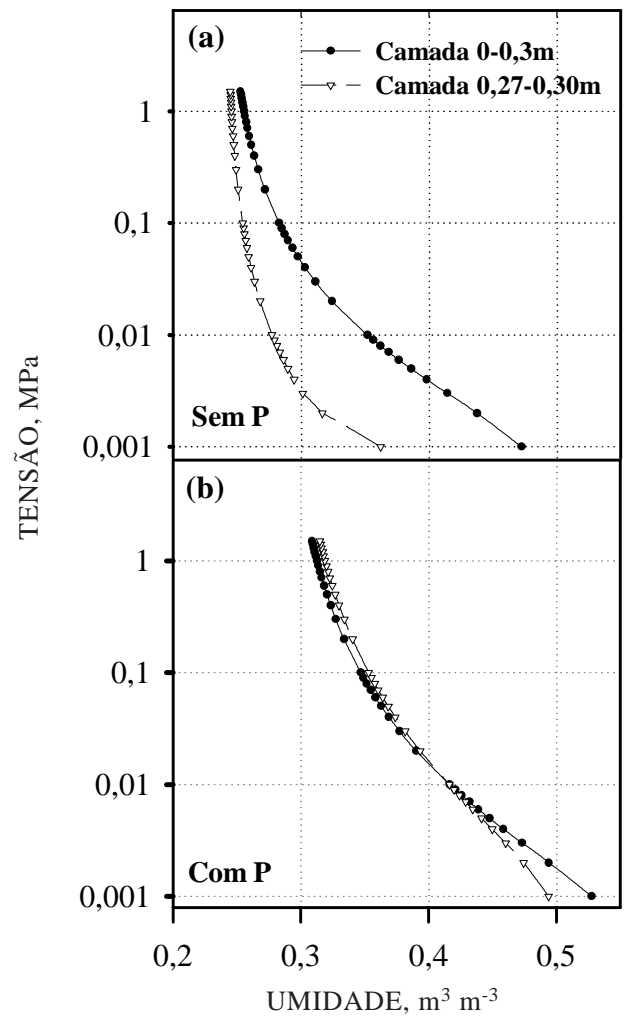

Figura 2. Curvas de retenção de água de material de solo, com e sem P, em duas camadas do solo.
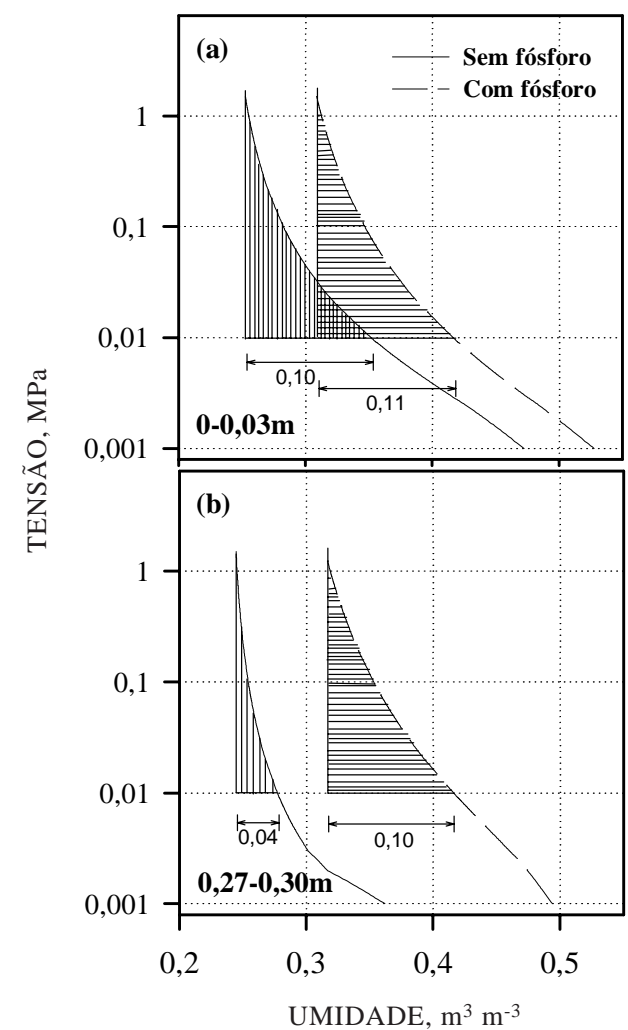

Figura 3. Curvas de retenção de água e os seus teores de água disponível, com e sem P, em duas camadas do solo.

Segundo Reichardt (1988), a capaci dadedecampo éresultado de um comportamento dinâmico da água no solo e não uma característica intrínseca da sua matriz. Portanto, alterações nas propriedades descritas neste estudo contribuíram para maior acréscimos do teor de água presente no solo em condições de ser absorvida pelas plantas.

\section{CONCLUSÕES}

1. A adsorção de $P$ em amostras de material de solo de um LVd el evou os teores de argila dispersa em água (ADA) e baixou o índice de floculação com conseqüente redução e aumento da macro e microporosidade, respectivamente; el evou os teores de água dos limites de liquidez, plástico, e de contração, provocando acréscimos do índice de plasticidade; influiu nos parâmetros de ajuste do modelo de Genuchten e deslocou as curvas de retenção de água para umidades mais el evadas.

2. A adsorção de $P$ influenciou a retenção deágua, nas tensões correspondentes à capacidade de campo e ponto de murcha permanente, elevando os teores de água disponível, principalmente na camada de $0,27-0,30 \mathrm{~m}$. 


\section{LITE RATURA CITADA}

ALVAREZ V., V.H. \& FONSECA, D.M. Definição de doses de fósforo para a determinação da capacidade máxima de adsorção de fosfatos e para ensaios em casa de vegetação. R. Bras. Ci. Solo, 14:49-55, 1990.

BAHIA FILHO, A.F.C. Índice de disponibilidade de fósforo em Latossolos do Planalto Central com diferentes características texturais e mineralógicas. Viçosa, Universidade Federal de Viçosa, 1982. 179p. (Tese de Doutorado)

BAHIA FILHO,A.F.C.;BRAGA,J .M.; RESENDE, M.\& RIBEIRO, A.C. Relações entre adsorção de fósforo e componentes mineralógicos da fração argila de Latossolos do Planalto Central. R. Bras. Ci. Solo, 7:221-226,1983.

BOWLES, J.A. Engineering properties of soils and their measurements. 3.ed. New York, McGraw-Hill, 1986. 218p.

CURI, N.; CAMARGO, O.A.; GUEDES, G.A.A. \& SILVEIRA, J.V. Sorção de fósforo em materiais de Latossolo do Brasil Sudeste. In: REUNIÃO DE CLASSIFICAÇÃO, CORRELAÇÃO DE SOLOS E INTERPRETAÇÃO DA APTIDÃO AGRÍ COLA, 3., Rio de J aneiro, 1988. Anais. Rio de J aneiro, Empresa Brasileira de Pesquisa Agropecuária, 1988. p.267-282.

DIAS J UNIOR, M.S. \& PIERCE, F.J . O processo de compactação do sol o e sua modelagem. R. Bras. Ci. Solo, 20:175-182, 1996.

DIAS J UNIOR, M.S. Compression of three soils under long-term tillage and wheel traffic. East Lansing, Michigan State University, 1994. 114p. (Tese de Doutorado)

DOURADO NETO, D.; LIER, Q.J .V.; BOTREL, T.A. \& LIBARDI, P.L. Programa para confecção da curva de retenção da água no solo utilizando o modelo Genuchten. Eng. Rural, 1:92$102,1990$.

EL-SWAIFY, S.A. \& EMERSON, W.W. Changes in the physical properties of soil clays due to precipited Aluminiun and iron hidroxides: I Swelling and aggregate stability after drying. Soil Sci. Soc. Am. Proc, 39:1056-1063, 1975.

EMPRESA BRASILEIRA DE PESQUISA AGROPECUÁRIA EMBRAPA - EMBRAPA. Centro Nacional de Pesquisa de Solos. Manual de métodos de análise desolo. Rio deJ aneiro, 1997. 212p.

GEE, G.W. \& BAUDER, J.W. Particle size analysis. KLUTE, A., ed. Methods of soils analysis. 2.ed. Madison, American Society of Agronomy, 1986. pt.1. p.383-411. (Agronomy Monography, 9)

GENUCHTEN, M.Th. A closed-form equation for predicting the conductivity of unsaturated soils. Soil Sci. Soc. Am. J., 44:892-898, 1980.

GI LLMAN, G.P. The influence of net charge on water dispersible clay and soberb sul phate. Aust.J . Soil Res, 12:173-176, 1974.

HINGSTON, F.J .; POSNER A.M. \& QUIRK.J .P. Anion adsorption by goethite and gibbsite. II: Desorption of anions from the hydrous oxide surfaces. J. Soil Sci., 25:16-26, 1974.
KENG,J .C. \& UEHARA, G. Chemistry, mineralogy and taxonomy of oxissols and Utisols. Proc. Soil Crop Sci. Soc. Florida, 33:119-126, 1974.

KLUTE, A. Laboratory measurement of hydraulic conductivity of saturated soils. In: KLUTE, A., ed. Methods of soil analysis. Madison, American Society of Agronomy, 1986. p.61-253. (Monography, 9)

KONDO, M.K. \& DIAS J UNIOR, M.S. Efeito do manejo e da umidade no comportamento compressivo de três Latossol os. R. Bras. Ci. Solo, 23:497-506, 1999.

KUO, S. \& LOTSE, E.G. Kinetics of phosphate adsorption and desorption by lake sediments. Soil Sci. Soc. Am. J., 38:5054, 1974.

LANDA, E.R. \& GAST, R.G. Evolution of crystallinity in hydrated ferrioxides. Clays Clay Miner., 21:121-130, 1973.

LIMA, J.M.; ANDERSON, S.J . \& CURI, N. Phosphate-induced clay dispersion as related to aggregate size and composition in hapludoxs. Soil Sci. Soc. Am. J., 64:892-897, 2000.

MEHRA, O.P. \& J ACKSON, M.L. I ron oxides removal from soils and clays by a dithionite-citrate-bicarbonate system buffered with sodium bicarbonate. Clays Clay Miner, 7:317327, 1960

MESQUITA FILHO, M.V. \& TORRENT, J. Phosphate sorption as related to mineralogy of a hidrosequence of soils form the Cerrado Region (Brazil). Geoderma, 58:107-123, 1993.

OLSEN, R.B. \& WATANABE, F.S. A method of determine a phosphorus adsorption maximum of soils as measured by the Langmuir isotherm. Soil Sci. Soc. Am. J ., 21:144-149, 1957.

PARFIT, R.L.; ATKINSON, R.J. \& SMART R.S.C. The mechanisms of phosphate fixation by iron oxides. Soil Sci. Soc. Am. J ., 39:837-841, 1975.

RAJ AN, S.S.S. \& FOX, R.L. Phosphate adsorption by soils: II.Reactions in tropical acid soils. Soil Sci. Soc. Am. Proc., 39:846-851, 1975.

RESENDE, M. Mineral ogy, chemistry, morphology and geomorphology of some soils of the Central Plateau of Brazil. West Lafayette, PurdueUniversity, 1976. 237p. (Tese de Doutorado).

REICHARDT, K. Capacidade de campo. R. Bras. Ci. Solo, 12:211216. 1988.

SAWHNEY, B.L. Charge characteristics of soils as affected by phosphate sorption. Soil Sci. Soc. Am. J ., 38:159-160, 1974.

SHAINBERG, I \& \& LETEY, J . Response of soil to sodic and saline conditions. J. Agric. Sci., 52:1-57, 1984.

SILVA, R.B.; LIMA, J.M. \& DIAS J UNIOR, M.S. Efeito da adsorção de fosfato em parâmetros físicos e na compressibilidade de solos tropicais. R. Bras. Ci. Solo, 23:219-226, 1999.

SOUZA, J.A.; CURI, N. \& GUEDES, G.A.A. Relação entre mineralogia e adsorção-desorção de fósforo em alguns Latossolos sob cerrado do Planalto Central. Ci. Prat. 15:103-111, 1991. 
SOWERS, G.W. Consistency. In: KLUTE, A., ed. Methods of soil analysis. 2. ed. Madison, American Society of Agronomy, 1986. p.391-399.

SPOSITO, G. Surface reactions in natural and aqueous colloidal systems. Chimia, 43: 169-176, 1989.

VALLE J UNIOR, R.F. Efeito da aplicação de hidróxido de sódio sobre a disponibilidade real de água de um Latossolo Vermel ho distrófico sob cerrado. Lavras, Universidade Federal de Lavras, 1995. 105p. (Tese de Mestrado)
VASCONCELLOS, A.C. Fósforo em dois latossolos do estado de Mato Grosso: adsorção, dessorção e crescimento vegetal. Viçosa, Universidade Federal de Viçosa, 1974. 103p. (Tese de Mestrado)

VEITH, J.A. \& SPOSITO, G. On the use of the Langmuir equation in the interpretation of adsorption phenomena. Soil Sci. Soc. Am. J ., 41:697-702, 1977.

VETTORI, L. Métodos de análise de solo. Brasília, Ministério da Agricultura, 1969. 24p. (Boletim Técnico, 7)

ZONTA, E.P.; MACHADO, A.A. \& SILVEIRA J ÚNIOR, P. Sistemas de análise estatística para microcomputadores (SANEST). Pelotas, Universidade Federal de Pelotas, 1984.151p. 